\title{
Progression and regression of fibrosis in viral hepatitis in the treatment era: the Beijing classification
}

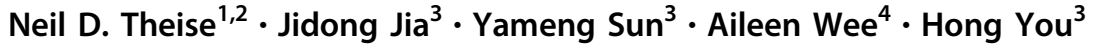

Received: 2 November 2017 / Revised: 23 January 2018 / Accepted: 10 February 2018 / Published online: 26 April 2018

(c) United States \& Canadian Academy of Pathology 2018

\begin{abstract}
In this new era of successful long term suppression of hepatitis B viral replication and consistent eradication of hepatitis C virus the necessity for routine pre-treatment biopsies has often been eliminated. Thus, whether there is utility to perform liver biopsy in chronic viral hepatitis is undergoing re-examination. In response to these changing needs, we have developed a new staging system, the Beijing Classification, for assessment of biopsy specimens from patients with chronic viral hepatitis. The most important novelty of the Beijing Classification is that it includes not only extent (stage) of fibrosis, but the quality of fibrosis, namely if the specimen shows predominantly regressive vs. progressive features (or is indeterminantly balanced between the two), the P-I-R score. This histologic distinction between regressive and progressive fibrosis, while invoked in this particular setting of chronic viral hepatitis, may have applicability to all forms of chronic liver disease. Thus, the review contains a description of the concepts of regression and progression with the aim of empowering pathologists to apply them in histopathologic-clinical correlation research as well as in the specific clinical setting for which it was developed. Also, in light of changing clinical needs, grading of necroinflammatory activity and staging of fibrosis are simplified into three point scales. These simplifications should aid the general diagnostic pathologist in being comfortable and confident in assessing biopsy specimens as the criteria for their distinction are far more precise, with significantly reduced "gray zones" of prior grading/staging systems.
\end{abstract}

\section{Introduction}

The potential for liver fibrosis to regress following successful treatment has been acknowledged for many years, particularly in the realm of viral hepatitis where successful suppression of hepatitis B virus (HBV) replication and eradication of hepatitis $\mathrm{C}$ virus (HCV) lead to regression of

Neil D. Theise

neil.theise@nyumc.org

1 Department of Pathology, Mount Sinai Beth Israel Medical Center, New York, NY, USA

2 Department of Pathology, New York University School of Medicine, New York, NY, USA

3 Liver Research Center, Beijing Key Laboratory of Translational Medicine in Liver Cirrhosis, National Clinical Research Center for Digestive Disease, Beijing Friendship Hospital, Capital Medical University, Beijing, China

4 Department of Pathology, Yong Loo Lin School of Medicine, National University of Singapore, National University Hospital, Singapore, Singapore scar in most patients [1-5]. However, incorporation of such concepts into diagnosis and assessment of liver diseases in biopsies and resection specimens has not kept pace. The original descriptive analysis of regressing fibrosis was produced by Wanless and colleagues [6] and while initially controversial [7-10], the ideas have gained acceptance in the community of pathologists academically focused on hepatopathology [11-14]. Regressing fibrosis in combination with concepts of vascular injury leading to parenchymal extinction are now understood to represent the dominant, tissue level pathways whereby scar progresses in viral hepatitis and contributes to progression in many other diseases as well.

In terms of viral hepatitis biopsies themselves, recent advances in the treatments of chronic viral hepatitis B and C have changed both the need for liver biopsy and the information expected from liver biopsy specimens when performed [1, 2, 15, 16]. Previously, establishment of baseline, pre-treatment fibrosis stage of disease was the most important feature for assessing both prognosis and the urgency of treatment needs [17]. However, with successful anti-viral therapies with low toxicity, the necessity for pre- 
treatment biopsies has often been eliminated $[15,16]$. Thus, the question of whether there is utility to perform liver biopsy in chronic viral hepatitis is undergoing reexamination.

Clearly, if there is clinical suspicion of concomitant diseases prior to treatment, biopsy still remains important. Furthermore, post-treatment biopsies may occasionally be necessary to assess the possibility of new or unexpected concomitant acute or chronic liver disease in the $90 \%$ of patients with successfully suppressed HBV replication and 95\% of cured HCV patients [18]. Also important, however, is the recognition that in some successfully treated viral hepatitis patients, there is no new disease; rather, the pretreatment injury to the liver is sufficiently severe-perhaps a combination of scarring and vascular alterations-that elimination of active viral infection does not allow the liver to successfully regress to earlier stages of disease.

Recently, we and our colleagues have attempted to address this question by studying pre-treatment and posttreatment biopsy specimens in patients with successfully suppressed HBV infection. Comparing both biopsy specimens to each other and correlating them with clinical outcomes, we proposed a new "Beijing classification" for evaluation of viral hepatitis liver biopsy specimens that may be of use in the coming years (Table 1) [19]. Uniquely, this classification incorporates histopathologic features of regressive vs. progressive fibrosis, i.e., the quality of fibrosis, not only its extent (Fig. 1). The importance of this finding is that it was predictive of outcomes; patients with predominantly regressive scarring were likely to do well post-treatment; those with predominantly progressive scarring or an "indeterminate" balance of regression/progression were likely to continue to progress their chronic liver disease despite successful treatment.

In reviewing this classification for practicing diagnostic pathologists who have liver pathology within their clinical purview, we not only wish to update them to possible important changes from the clinical side of viral hepatitis management, but we also suggest the utility of the Beijing Classification to assessment and reporting of regressive/ progressive fibrosis for studies of clinical-pathologic correlation in all forms of chronic liver disease, more generally.

\section{The Beijing Classification}

To arrive at the new classification, we analyzed histologic changes in pre-treatment and post-treatment liver biopsy specimens in 71 patients with successfully treated HBV infection. Routine stains used in the study were hematoxylin and eosin (H\&E), Masson trichrome, and reticulins. Traditional grading of necroinflammatory activity and staging of fibrosis were analyzed according to the Ishak
Scoring System [20]. Patients with stage 5 and 6 fibrosis ("cirrhosis") were further assessed according to the Laennec staging system [21, 22]. Additionally, automated two photon excitation fluorescence-second harmonic generation analysis of collagen in all biopsy specimens was performed, though this technique, confirming fibrotic staging [23], did not alter assessment of specimens from the point of the view of the final classification [24]. Thus, all Beijing Classification parameters could be performed on routine traditionally prepared liver biopsy slides. It was also found that reticulin and trichrome stains were interchangeable for assessment of all aspects of fibrosis; thus, whichever stain is most consistently performed in any given diagnostic laboratory can be used for classification.

The chief novelty of the Beijing Classification, as noted, is that in addition to the traditional grading of necroinflammatory activity and staging of fibrosis we recognized that the quality of fibrosis was a separate, independent feature that gave valuable clinical information, correlating to post-treatment outcomes. The qualities recognized in the analysis correspond to those first described by Wanless et al. [6] specifying histologic features that reflect regression of fibrosis rather than active, progressing scar. Given the value of this new parameter and the lesser importance of detailed distinction of degrees of necroinflammation and fibrotic stage, we then suggested simplifications of the traditional methods of assessment. As noted in Table 1, these are simplifications for daily clinical use; they may be substituted with more complex staging systems (e.g., Ishak, Metavir) for research purposes or in response to needs and interests of the clinicians [17]. However, we feel that these simplifications allow the diagnostic pathologist and their clinical colleagues to clearly focus on the most important clinical correlations in the most direct fashion possible.

\section{P-I-R score}

While this part of the classification is stated as a third, additional, and supplemental feature, we begin this review with its discussion given its novelty in the practice of liver pathology. It is based primarily on the work of Ian Wanless and colleagues in which the regression of cirrhosis was highlighted, first in treated chronic hepatitis B and then in other studies and in other diseases [7]. While the possibility that cirrhosis could regress to livers with improved or even normal functioning status was highly controversial when Wanless first presented the concept [7-10], subsequent reports, particularly in patients with hepatitis $\mathrm{C}$ cirrhosis in which sustained viral response was obtained by interferonbased therapies, confirmed that this actually happens [3-5].

A simple statement of Wanless' fairly complex understanding of the progression and regression of liver disease, as it applies to chronic viral hepatitis, emphasizes vascular 
Table 1 Beijing Classification for histologic assessment of chronic viral hepatitis

\begin{tabular}{|c|c|c|}
\hline Hepatitis assessment: & Description & Prior classifications \\
\hline Inactive & $\begin{array}{l}\text { Portal inflammation only or rare foci of interface or } \\
\text { lobular hepatitis; no confluent necrosis }\end{array}$ & $\begin{array}{l}\text { Chronic persistent hepatitis } \\
\text { Ishak HAI } 1-5 \\
\text { Metavir A1 } \\
\text { Batts-Ludwig grade } 1\end{array}$ \\
\hline Active, non-severe & $\begin{array}{l}\text { Varying degrees of interface and lobular hepatitis } \\
\text { easily identified at low power; no confluent necrosis }\end{array}$ & $\begin{array}{l}\text { Chronic active (aggressive) hepatitis } \\
\text { Ishak HAI 5-12 } \\
\text { Metavir A1-A2 } \\
\text { Batts-Ludwig grade 2-3 }\end{array}$ \\
\hline Active, severe ${ }^{a}$ & $\begin{array}{l}\text { Confluent necrosis (perivenular drop out or bridging } \\
\text { necrosis or parenchymal collapse) } \\
\text { NOTE: This definition of severe activity raises the } \\
\text { question of possible concomitant diseases (e.g., AIH, } \\
\text { DILI) or immunosuppression (e.g., untreated HIV). }\end{array}$ & $\begin{array}{l}\text { Chronic active (aggressive) hepatitis } \\
\text { Ishak HAI 13-18 } \\
\text { Metavir A3 } \\
\text { Batts-Ludwig grade } 4\end{array}$ \\
\hline \multicolumn{3}{|l|}{ Fibrosis stage } \\
\hline Early & No fibrosis or portal fibrosis & $\begin{array}{l}\text { Ishak stage } 0-2 \\
\text { Metavir F0-1 } \\
\text { Batts-Ludwig stage } 0-2\end{array}$ \\
\hline Intermediate & Fibrous septa, focal or frequent & $\begin{array}{l}\text { Ishak stage } 3-4 \\
\text { Metavir F2-F3 } \\
\text { Batts-Ludwig stage } 3\end{array}$ \\
\hline Advanced & $\begin{array}{l}\text { Fibrous septa with focal or diffuse nodularity } \\
\text { (developing or established "cirrhosis") }\end{array}$ & $\begin{array}{l}\text { Ishak stage } 5-6 \\
\text { Metavir F3-F4 } \\
\text { Batts-Ludwig stage } 3-4\end{array}$ \\
\hline \multicolumn{3}{|l|}{ P-I-R fibrosis quality } \\
\hline $\begin{array}{l}\text { Predominantly } \\
\text { Progressive features }\end{array}$ & Most of specimen shows progressive forms of stroma & Laennec $4 \mathrm{~A}^{\mathrm{b}}$ or $4 \mathrm{~B}$ or $4 \mathrm{C}$ \\
\hline Indeterminate & $\begin{array}{l}\text { Uncertain mix/balance between progressive and } \\
\text { regressive stroma }\end{array}$ & Laennec 4B \\
\hline $\begin{array}{l}\text { Predominantly } \\
\text { Regressive features }\end{array}$ & Most of specimens shows regressive forms of stroma & Laennec 4A \\
\hline Not applicable & $\begin{array}{l}\text { Not used in biopsies with "early stage" fibrosis, i.e., } \\
\text { without fibrous septa }\end{array}$ & \\
\hline
\end{tabular}

${ }^{a}$ When severe (confluent) necrosis is present in biopsy specimens from patients with viral hepatitis, it indicates the need for clinical exclusion of concomitant autoimmune hepatitis, drug/toxin induced liver injury, co-infection with other hepatotropic viruses, or immunosuppression (e.g., HIV co-infection)

${ }^{\mathrm{b}}$ Published illustration of Laennec 4A shows predominantly regressive features, but progressive 4A may also be identified

injuries that follow on the portal and parenchymal inflammation of viral infection (Fig. 2). He describes zones of parenchymal extinction spanning areas of the hepatic lobule in which there is obstruction to vascular inflow (usually portal vein) and outflow (hepatic venules and veins) secondary to the thrombosis instigated by inflammation. Such zones of parenchymal extinction span the occluded vessels leading to further inflammatory changes, activation of hepatic stellate cells [25], collapse of reticulin meshwork [26], and ductular reactions that are activations of hepatobiliary stem cell niches [27]. These lesions, in the presence of continuing viral replication and virus-associated inflammation, may also, to some extent be self-propagating and perpetuating as liver architecture distorts over the years of chronic disease.
When the viral infection is suppressed or eliminated, features of regression often begin to predominate. Wanless named these histologic findings the hepatic repair complex (Table 2), eight features reflecting three types of tissue responses: 1. changes in the qualities and distribution of scar; 2. persistent vascular distortions following on the vascular injuries that lead to scarring in the first place; and 3. hepatocellular regeneration [7]. During the course of active disease these features are often present admixed with the active, progressing lesions. Indeed, Wanless has emphasized that there is a seesaw affect in chronic disease: while the overall direction of changes reflects progression, the liver is also, always, making attempts to repair the lesions. However, in the absence of disease remission it is a losing battle. 


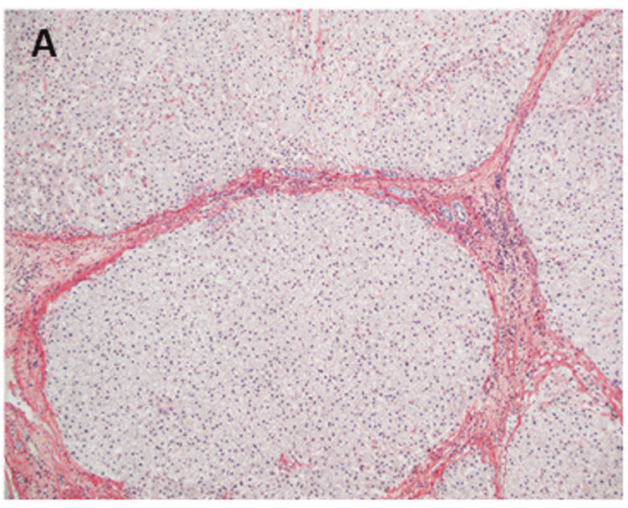

Fig. 1 Progressive vs. regressive septa in advanced stage liver disease. In an advanced stage (cirrhotic) liver secondary to chronic hepatitis B (tissue obtained at hepatectomy for hepatocellular carcinoma), tissue is stained with Sirius red stain to highlight differences between progressive and regressive fibrous septa. a The broad, progressive septa show loosely aggregated, pale staining collagen, edema, congestion, and inflammatory cells. (Sirius red, 10x) b. The thin, regressive septa

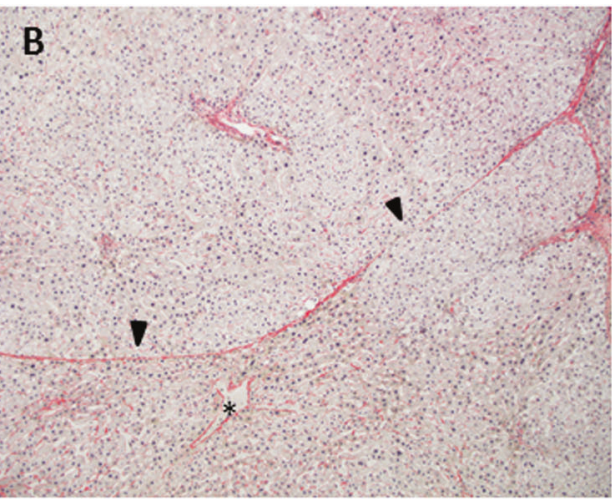

show densely compacted, richly staining collagen-without edema, inflammatory or other cellular infiltrates-and foci of disintegration of scar where hepatocytes interrupt the scar (arrow heads). Asterisk marks a probable central venule in too close proximity to the septum typical of vascular distortions of advanced stage disease. (Sirius red, 10x)
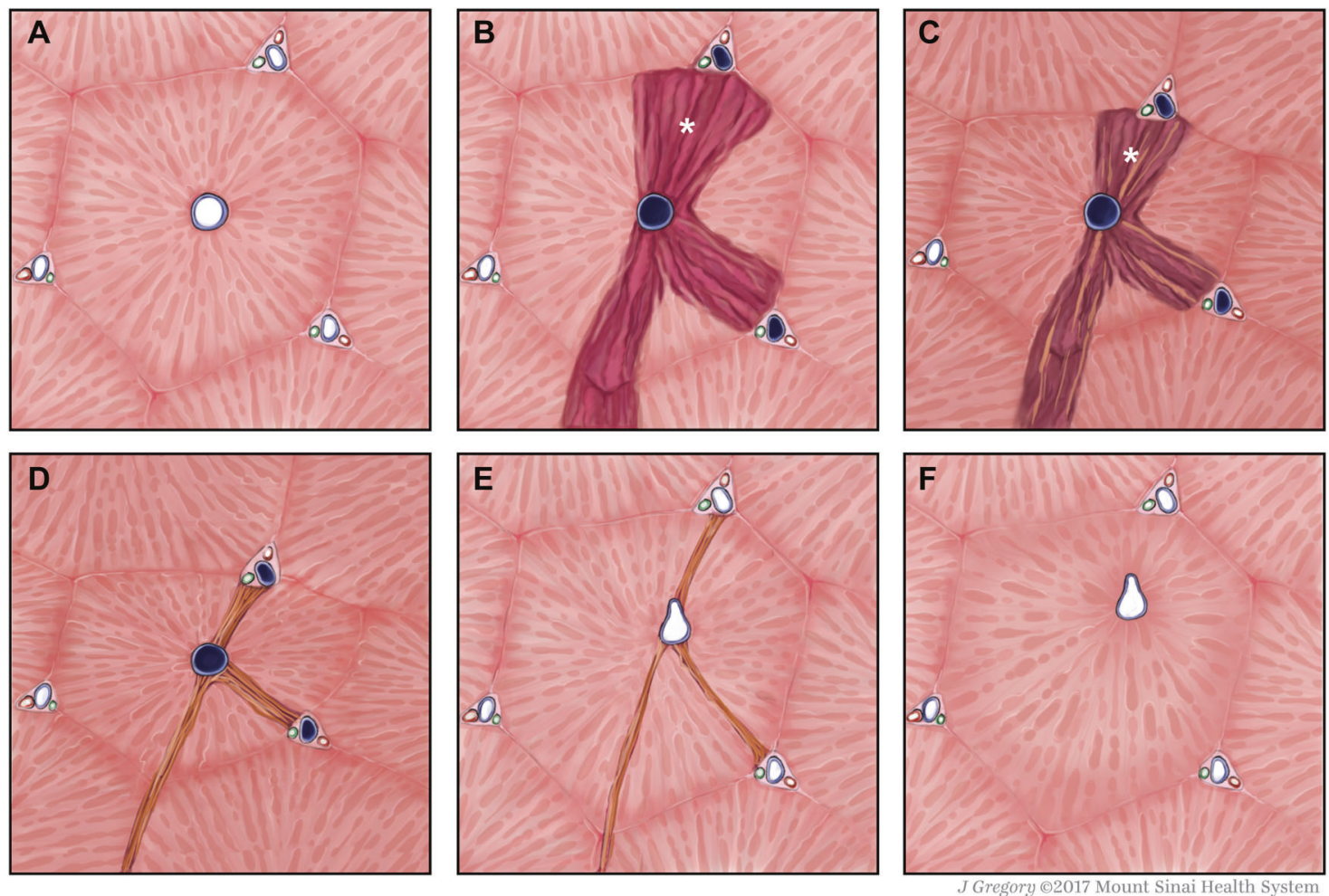

Fig. 2 Progression of chronic hepatitis liver disease. a Schematic of normal hepatic parenchyma. b Early parenchymal extention (*; redbrown) forms when there are vascular thrombosis to two vessels of hepatic parenchymal blood supply (portal veins and central vein here; black indicating occlusion). Early parenchymal extinction is marked by inflammation, macrophages, activation of hepatic stellate cells, minimal collagen deposition. $\mathbf{c}$ Progressing parenchymal extinction (*; dark brown) has increasing deposition of collagen with broad fibrous septa. These are variably inflamed. Hepatic stellate cells remain prominent. Ductular reactions may be present. d With quiescent disease, fibrotic septa are dense and well formed. Inflammation is less. Stellate cells may still be active, though often less prominently. e Regressing septa are densely compacted, acellular. There may be fragmentation. Vascular thrombosis may be demonstrably recanalized. Elastin fibers are prominent with special stains. F. Regression may be complete in some cases, though distortion of parenchymal anatomy may be recognizable, central veins and portal tracts in abnormal proximity 
We applied a simple three-point semi-quantitative assessment for evaluating this balance between progression and regression, rating each specimen as "predominantly progressive", "predominately regressive" or "indeterminate" (Fig. 3). This assessment was made at low power, using either trichrome or reticulin stain (depending on the quality of the stains in any given case). Sirius red stain is equivalently useful (Fig. 1). Most cases were easy to define as predominantly progressive or regressive at a glance, scanning along the slide at low power. Indeterminate cases are perhaps best described as those for which the pathologist literally cannot decide, despite continuing examination of the slide. Also in these cases, there was a temptation to go to high power for better assessment- though such higher power assessment only helped one evaluate the quality of foci of fibrosis, but not the overall balance in the specimen. This is the most important "helpful hint" for application of this P-I-R scoring: the pathologist should not worry about

Table 2 Hepatic repair complex

\begin{tabular}{ll}
\hline & $\begin{array}{l}\text { Histological features for } \\
\text { regression }\end{array}$ \\
\hline $\begin{array}{l}\text { Fragmentation and regression of } \\
\text { scar }\end{array}$ & $\begin{array}{l}\text { Delicate, perforated septa } \\
\text { Isolated thick collagen fibres } \\
\text { Delicate, periportal fibrous } \\
\text { spikes }\end{array}$ \\
& $\begin{array}{l}\text { Hepatocytes within or splitting } \\
\text { septa } \\
\text { Portal tract remnants } \\
\text { Evidence of prior, now resolving, } \\
\text { vascular derangements }\end{array}$ \\
& $\begin{array}{l}\text { prolapsed hepatocytes } \\
\text { Aberrant parenchymal veins } \\
\text { Hepatocyte buds }\end{array}$ \\
\hline
\end{tabular}

the score: if it is not obviously one or the other, then it is simply "indeterminate."

The simplicity, even perhaps naiveté of such an approach may be off putting to the pathologist seeking a true quantification. But as every pathologist who has had to deal with grading/staging of viral hepatitis liver biopsy specimens knows, there is no perfect system; simple systems of semiquantification are more reproducible, by a single pathologist on repeat examinations or by multiple pathologists in interobserver studies [28, 29]. Thus, for example, the Metavir system was developed to maximize the Kappa statistics reflecting inter-observer agreement and, not surprisingly, the four stages of fibrosis and three grades of necroinflammation are more reproducible than the more detailed and complete Ishak grading and staging system [30]. Indeed, Kappa statistics for this P-I-R staging (performed in the same manner as that reported for the development of Metavir) showed a striking consistency: Kappa value of 0.71 ("substantial agreement"), essentially equivalent to the original Metavir analysis. Furthermore the 12 participants in that part of the study were specialized liver pathologists, general diagnostic pathologists, clinical hepatologists and one engineer, emphasizing that the classification will be easily and consistently applied in all diagnostic settings in which patients with viral hepatitis are being treated [19].

The value of the P-I-R scoring was revealed by their associated clinical correlations. In this first published study, it dynamically reflected the prognosis of the disease, independent of inflammation activity, fibrosis severity, or treatment experience. It also extended the conventional definition of "reversal of fibrosis" from decreasing $>=1$ stage by Ishak system, to $\mathrm{P} / \mathrm{I}$ before treatment to $\mathrm{R}$ after treatment. Further validation studies are needed and encouraged to both confirm these findings and to evaluate

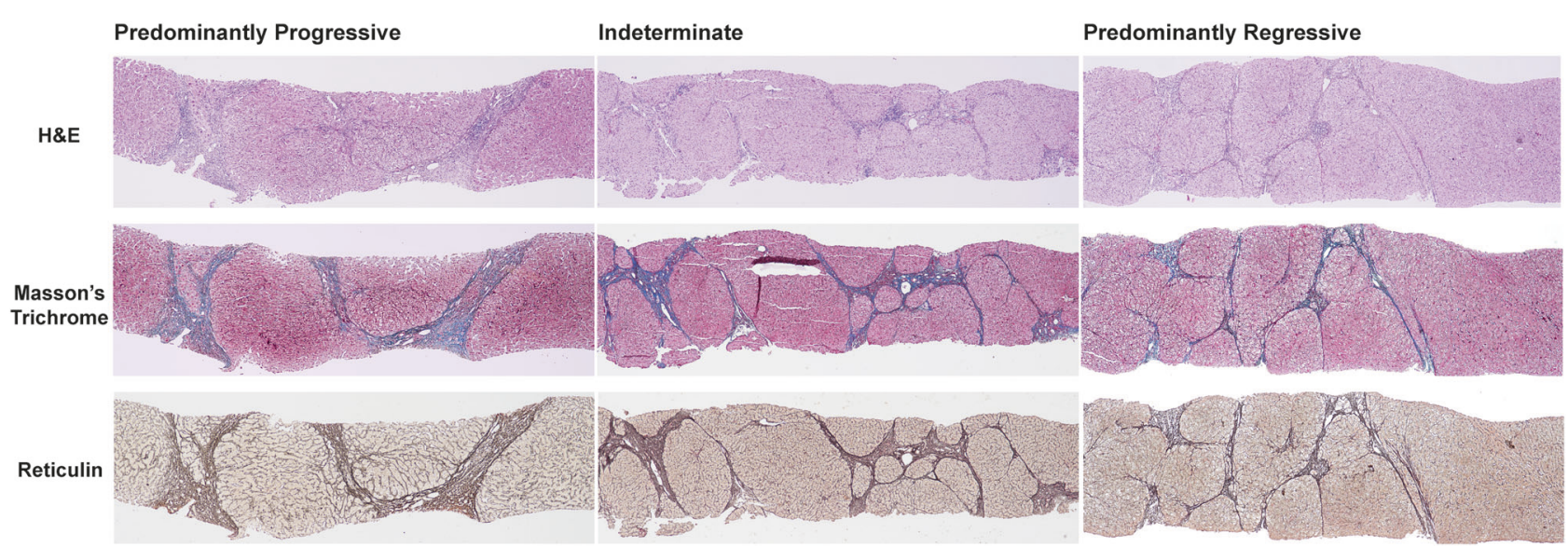

Fig. 3 The new fibrosis quality classification (P-I-R score). The morphologic balance between progressive and regressive of fibrosis/scarring. $(2 x)$ There are three classification categories: predominantly progressive (a), indeterminate (b), and predominately regressive (c). $\mathrm{H} \& \mathrm{E}$ hematoxylin and eosin 
the potential for extended utility of the P-I-R scoring in different disease settings.

Thus, in this era of successful viral treatment, it seems that a post-treatment biopsy, in addition to or even perhaps more than the pre-treatment biopsy, will serve the clinical needs of patients and their clinicians. In this regard, one extra point is worth noting: one patient in our study population began active alcohol use in the post-treatment period-thus, the most important finding in that biopsy specimen was evidence for a new and unsuspected liver disease requiring attention. As previously mentioned, concomitant or additional diseases are sometimes clinically concealed by expectations regarding the known liver disease and only a biopsy can lead to the important clinical occurrence of a new, superimposed disease. We will return to this issue in our discussion of necroinflammatory activity.

\section{Grading of necroinflammation}

The earliest grading system, from the "International Study Group" described chronic persistent hepatitis ( $\mathrm{CPH}$ ) and chronic active (or aggressive) hepatitis (CAH) [31]. These were first proposed, in fact, in response to the discovery of HBV in 1968, when a majority of "post-transfusional chronic hepatitis" cases were found to be caused by this single agent [32]. This was the simplest possible semiquantification. It was superseded by the same group, spearheaded by member Kamal Ishak and his colleague R. G. Knodell, in what became known in its final form as the Ishak system [33]. This detailed analysis encompassed four patterns of inflammation (portal, piecemeal necrosis-now generally referred to as interface hepatitis, lobular hepatitis and confluent necrosis) in a range of possible scores from 1 to 18 . Of note is that confluent necrosis, comprising six possible points (from focal to perivenular to bridging necrosis to pan- or multiacinar collapse), is necessary to achieve a "severe" grade of activity (Fig. 4).

Application of this system was certainly laborious, however, and best suited to the original intentions of the Ishak system: research. However, the number of details also meant inter-observer variation was likely to be greater and a simpler, more reproducible system was needed. The Metavir grading of activity accomplished this with an algorithmic approach comprising three grades, A1 to A3 [34]. This simplicity led to greater reproducibility and improved inter-observer variation [30].

Despite this improvement, the prognostic aspects of this grading of activity increasingly became almost incidental. The continued expectation of such assessment reflected, to some degree, the history going back to $\mathrm{CPH}$ vs. CAH. However, increasingly they were actually little used in the decision to treat or not to treat a patient with chronic viral hepatitis. Fibrosis stage was more often the deciding factor.
The differences between Metavir A1 and A2 or Ishak HAI 1-12 were of little clinical importance; likewise for the mild to moderate activity grades of the popular Batts-Ludwig classification [35].

However, the most severe activity, reflected very specifically in "confluent necrosis" described by the Ishak system, still has great clinical importance [20,33]. While such severe activity may reflect the activity of the virus itself (e.g., viral hepatitis "acute flare" or serologic conversion from hepatitis B e antigen to e antibody titers), it is often a sign of other, concomitant diseases [18]. For example, confluent necrosis may be the first clue to immunosuppression, particularly from untreated HIV (perhaps unsuspected by the clinical team), concomitant de novo autoimmune hepatitis, or drug/toxin induced liver injury. All of these are significant events, potentially signaled only by the presence of confluent necrosis, requiring particular therapeutic interventions.

In the Beijing Classification we have simplified the grading of activity to "inactive", "active, non-severe", and "active, severe." As with P-I-R scoring and Metavir activity scores, the three-point system is expected to promote good intra-observer and inter-observer agreement (not yet formally tested, however). The first two are analogous to $\mathrm{CPH}$ and $\mathrm{CAH}$ - they require little effort by the general pathologist. To our knowledge no further subdivision of these has any clinical implications. The third category implies confluent necrosis - this is the most important aspect of activity for the diagnostic pathologist to recognize, because it may signal concomitant or new liver disease requiring clinical attention (Fig. 5).

\section{Staging of fibrosis}

The Beijing Classification modification of staging again reflects the need for simplicity and the fact that detailed fibrosis stage is no longer decisive in deciding whether a patient will be treated. In the absence of that clinical need, a simpler scheme, reflecting the broad areas of clinical stage of disease is best applied. Again, a three-point system allows for strong agreement. The "early" stage corresponds to Ishak stages 1 and 2, Metavir F1 and Batts-Ludwig stage 1: some amount of portal fibrosis; no fibrous septa. The "intermediate" stage corresponds to Ishak stages 3 and 4, Metavir F2 and F3, and Batts-Ludwig stage 2: focal or frequent fibrous septa. In this new clinical context these stages (focal vs. frequent septa) can be grouped since the extent of septum formation is less important than the fibrosis quality so these stages; whether focal or frequent, they are unlikely, post-treatment, to progress to more advanced disease (though this is an assumption that remains unstudied). The third "advanced" stage corresponds to Ishak stages 5 and 6, Metavir F4, and Batts-Ludwig stages 3 and 
PREDOMINANTLY PROGRESSIVE (P): The ratio of progressive septa to regressive septa is clearly high

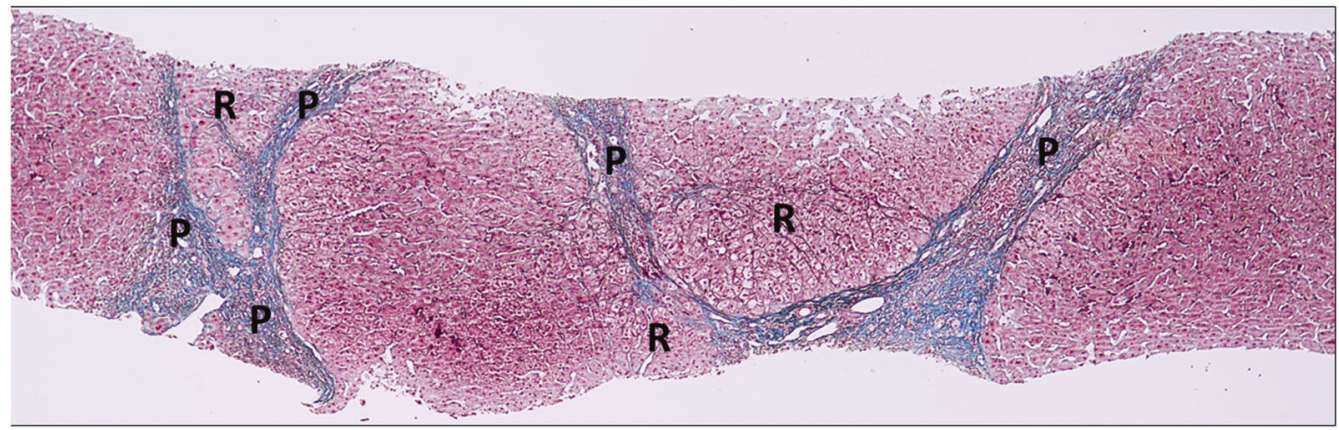

INDETERMINATE (I): Ratio of regression and progression appear balanced, neither one clearly predominating

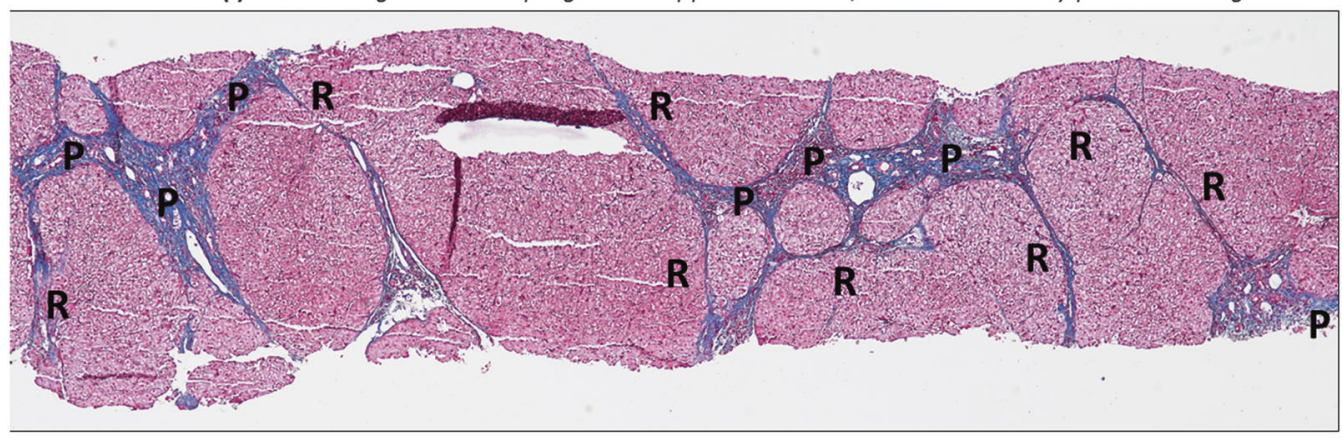

PREDOMINANTLY REGRESSIVE (R): The ratio of regressive septa to progressive septa is clearly high

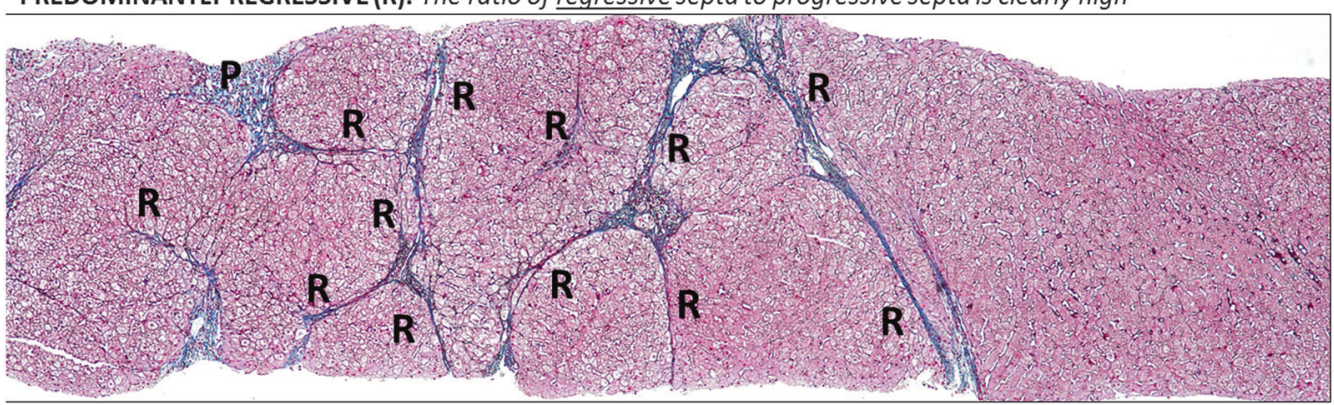

Fig. 4 Highlighted views of P-I-R liver biopsy findings. The right hand panels from Fig. 3 are expanded and annotated indicating progressive (P) vs. regressive (R) septa. The ratio of progressive to regressive is clearly large in the top panel which is therefore graded "predominantly

4 , reflecting what has historically been called "developing cirrhosis" and "established cirrhosis."

In calling it "advanced stage" viral hepatitis we have chosen to follow the recommendations of the International Liver Pathology Study Group which has recognized that the word cirrhosis has unintended associations (particularly that it is almost inevitably fatal without liver transplantation) and that cirrhoses of different diseases truly differ from each other morphologically and clinically [36, 37]. One word does not fit all. And now with the demonstration that cirrhosis can regress, a less rigid and definitive term best serves clinical needs. So we suggest "advanced stage" disease. progressive." The ratio is reversed, with regression predominating over regression in the lower panel yielding a grade of "predominantly regressive." The middle panel does not show overall predominance of one over the other, thus it is graded "indeterminate."

Again, as with activity grading, for research purposes, any of the other prior systems may be substituted for the Beijing fibrosis stage. But for general clinical practice and care of an individual patient, this simplified system should, we believe, prove easily applicable and consistently useful. One particular note in its usage needs to be pointed out: in the absence of fibrous septa (i.e., in early stage disease), there is no P-I-R score as the criteria for progressive vs. regressive scarring only follow on parenchymal extinction and their sequelae.

It is also interesting to then review the other more recent clinical staging system that has been suggested, that of the Laennec staging, a four point staging system in which stage 

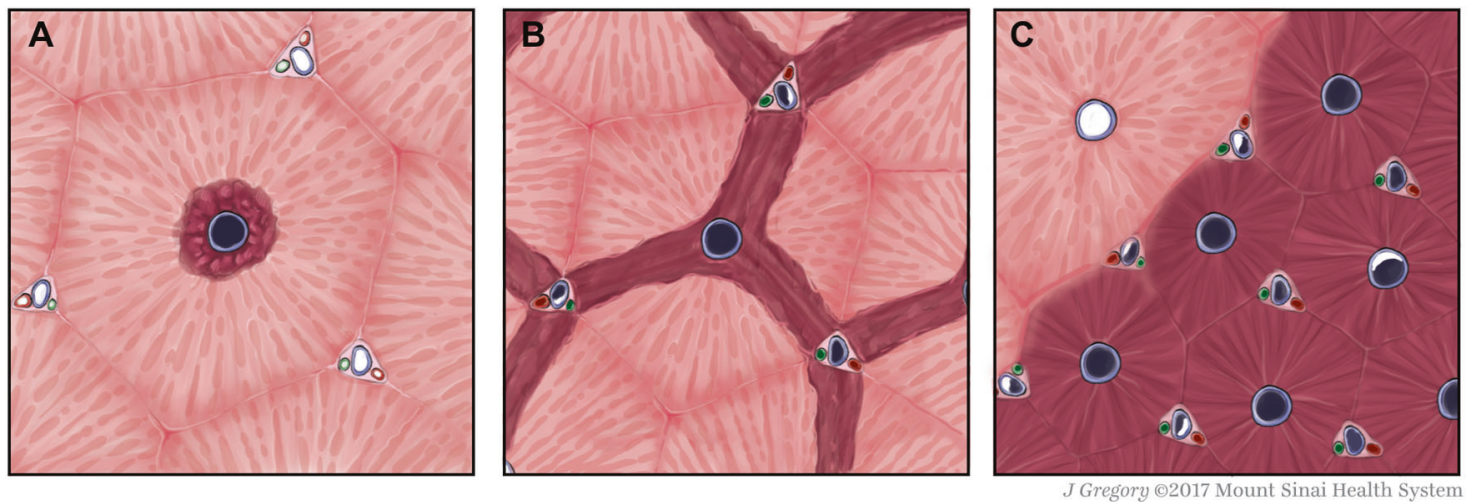

Fig. 5 Confluent necrosis. The principle hallmark of severe necroinflammatory activity is confluent necrosis which may be perivenular (a), bridging between portal tracts and/or between central veins and portal tracts (b), or so widespread there are regions of parenchymal

4 ("cirrhosis") is subdivided into stages 4A, 4B, and 4C reflecting the ratio of connective tissue to hepatic parenchyma in the specimen [21,22]. The primary study using that system clearly demonstrated that these stages correlated well with hepatic venous wedge pressures (HVWP). HVWP is perhaps the best method of determining prognosis and need for clinical interventions, following on chronic liver disease, in particular portal hypertension [38]. However, a review of that paper shows that stage 4A actually represents "predominantly regressive" fibrosis [39]! So while the implicit understanding of those authors indicates a sequence of development from $4 \mathrm{~A}$ to $4 \mathrm{~B}$ to $4 \mathrm{C}$, in all likelihood it represents the sequence of $4 \mathrm{~B}$ to $4 \mathrm{C}$ and, if viral activity spontaneously remits or is successfuly treated, either of these may evolve to $4 \mathrm{~A}$.

While this complicates the use of the Laennec system, it suggests a stronger utility for the Beijing Classification, namely that in advanced stage viral hepatitis $\mathrm{P}$ vs I vs R is a surrogate for clinical outcomes dependent on HVWP measurements. Given that the liver biopsy is a widespread practice and HVWP is still significantly invasive and still only performed in very specialized institutions, the combination of fibrosis stage and fibrosis quality scores of the Beijing Classification may suffice, providing similar prognostic information to HVWP for institutions lacking that test.

\section{Conclusion}

In summary, we have developed a new stage system for assessment of biopsy specimens from patients in chronic viral hepatitis. It may be applied to pre-treatment or posttreatment biopsies, but the most significant information may be derived from the post-treatment specimens; thus, one collapse (c); note that the schematic is drawn to same scale as a and $\mathbf{b}$, but structures have collapsed together as hepatic parenchyma is destroyed

implication of the new system may be a new approach concerning when to perform protocol biopsies. Further studies by other groups and institutions will be needed to refine questions of biopsy timing and the prognostic utility of the information.

The simplified system for activity grading and fibrosis staging should improve reliability even by the most general pathologists who have infrequent exposure to liver biopsy analysis. The highest grade of activity, confluent necrosis, has clear and important clinical implications. The highest "advanced" fibrosis stage likewise has important clinical implications: these are the patients that still have a risk, however small, of continuing to progress to continuing liver disease despite viral suppression [40]. They also perhaps have the greatest risk for development of hepatocellular carcinoma (though other stages also still have risk); the most useful aspect of this stage assignment may be to encourage the patient to comply with long term screening for malignancy [40-44].

However, the relatively small percentage of patients with advanced stage disease who continue to progress despite treatment means that simply stating "advanced stage" is not particularly useful. For this evaluation we add the P-I-R score for the quality of fibrosis indicating continuing progressive disease (inclusive of $\mathrm{P}$ and $\mathrm{I}$ scores) as opposed to those who are predominantly progressive. Such livers have reached a stage of self-perpetuating, but virus-independent vascular injury and thrombosis. These patients may require shunts or transplantation even though the treatment has been "successful." The P-I-R score in patients with advanced stage disease may also be a surrogate marker for HVWP at institutions that do not yet perform that test.

These are all preliminary thoughts on a newly proposed system. As with each of the systems that came before, a 
great variety of studies and approaches to their application, by many research groups, in many populations, will flesh out its utility and how best to apply it. We look forward to the possible development of applications of this concept of progressive vs. regressive qualities of fibrosis to other liver disease, in particular alcoholic and non-alcoholic fatty liver disease, as anti-fibrogenic treatments begin to move toward clinical use.

Acknowledgements We are grateful to Jill Gregory (Mt. Sinai Health System) for illustrations.

\section{Compliance with ethical standards}

Conflict of interest Dr. Theise has received consulting fees and travel support from Choutu Industries. The other authors declare that they have no conflict of interest.

\section{References}

1. Marcellin P, Gane E, Buti M, et al. Regression of cirrhosis during treatment with tenofovir disoproxil fumarate for chronic hepatitis B: a 5-year open-label follow-up study. Lancet. 2013;381: 468-75.

2. Chang TT, Liaw YF, Wu SS, et al. Long-term entecavir therapy results in the reversal of fibrosis/cirrhosis and continued histological improvement in patients with chronic hepatitis B. Hepatology. 2010;52:886-93.

3. Poynard T, McHutchison J, Manns M, et al. Impact of pegylated interferon alfa- $2 \mathrm{~b}$ and ribavirin on liver fibrosis in patients with chronic hepatitis C. Gastroenterology. 2002;122:1303-13.

4. Pockros PJ, Hamzeh FM, Martin P, et al. Histologic outcomes in hepatitis $\mathrm{C}$-infected patients with varying degrees of virologic response to interferon-based treatments. Hepatology. 2010;52:1193-1200.

5. Manne V, Akhtar E, Saab S. Cirrhosis regression in patients with viral hepatitis B and C: a systematic review. J Clin Gastroenterol. 2014;48:e76-84.

6. Wanless IR, Nakashima E, Sherman M. Regression of human cirrhosis. Morphologic features and the genesis of incomplete septal cirrhosis. Arch Pathol Lab Med. 2000;124:1599-607.

7. Ray MB. Regression of cirrhosis. A timely topic. Arch Pathol Lab Med. 2000;124:1589-90.

8. Chedid A. Regression of human cirrhosis. Arch Pathol Lab Med. 2000;124:1591.

9. Chejfec G. Controversies in pathology. Is cirrhosis of the liver a reversible disease? Arch Pathol Lab Med. 2000;124:1585-6.

10. Geller SA. Coming or going? What is cirrhosis? Arch Pathol Lab Med. 2000;124:1587-8.

11. Crawford JM. Cirrhosis. In: Odze RD, Goldblum JR, eds. Surgical pathology of the GI Tract, Liver, Biliary Tract, and Pancreas. 3rd edn. Philadelphia, Saunders Elsevier.

12. Sempoux C, Guido M, Saxena R. Cirrhosis: a term in need of a makeover. In: Saxena R, ed. Practical Hepatic Pathology: A Diagnostic Approach. 2nd edn. Philadelphia, Elsevier.

13. Theise ND. Liver and gallbladder. In: Kumar V, Abbas AK, Aster JC, eds. Robbins and Cotran Pathologic Basis of Disease. 9th edn. Philadelphia, Saunders Elsevier.

14. Crawford JM, Burt AD. Anatomy, pathophysiology and basic mechanisms of disease. In: Burt AD, Portman BC, Ferrell LD, eds. MacSween's Pathology of the Liver. 6th edn. London, Churchill Livingston Elsevier.
15. European Association for the Study of the Liver. EASL 2017 Clinical Practice Guidelines on the management of hepatitis B virus infection. J Hepatol. 2017. https://doi.org/10.1016/j.jhep. 2017.03.021.

16. European Association for the Study of the Liver. EASL recommendations on treatment of hepatitis C 2016. J Hepatol. 2017;66:153-94.

17. Theise ND. Liver biopsy assessment in chronic viral hepatitis: a personal, practical approach. Mod Pathol. 2007;20(Suppl 1): S3-14.

18. Hudacko R, Theise N. Liver biopsies in chronic viral hepatitis: beyond grading and staging. Arch Pathol Lab Med. 2011;135:1320-8.

19. Sun Y, Zhou J, Wang L, et al. New classification of liver biopsy assessment for fibrosis in chronic hepatitis B patients before and after treatment. Hepatology. 2017;65:1438-50.

20. Ishak K, Baptista A, Bianchi L, et al. Histological grading and staging of chronic hepatitis. J Hepatol. 1995;22:696-9.

21. Kim MY, Cho MY, Baik SK, et al. Histological subclassification of cirrhosis using the Laennec fibrosis scoring system correlates with clinical stage and grade of portal hypertension. J Hepatol. 2011;55:1004-9.

22. Kim SU, Oh HJ, Wanless IR, et al. The Laennec staging system for histological sub-classification of cirrhosis is useful for stratification of prognosis in patients with liver cirrhosis. J Hepatol. 2012;57:556-63.

23. Xu S, Wang Y, Tai DC, et al. qFibrosis: a fully-quantitative innovative method incorporating histological features to facilitate accurate fibrosis scoring in animal model and chronic hepatitis B patients. J Hepatol. 2014;61:260-9.

24. Wang Y, Vincent R, Yang J, et al. Dual-photon microscopy-based quantitation of fibrosis-related parameters ( $\mathrm{q}-\mathrm{FP}$ ) to model disease progression in steatohepatitis. Hepatology. 2017;65:1891-903.

25. Tsuchida T, Friedman SL. Mechanisms of hepatic stellate cell activation. Nat Rev Gastroenterol Hepatol. 2017;14:397-411.

26. Burt AD, Portmann BC, Ferrell LD. MacSween's Pathology of the Liver. In: Theise ND, Bodenheimer Jr HC, Ferrell LD, eds. Acute and chronic viral hepatitis. 6th edn. Edinburgh: Elsevier; 2012. pp. 361-93.

27. Gouw AS, Clouston AD, Theise ND. Ductular reactions in human liver: diversity at the interface. Hepatology. 2011;54:1853-63.

28. Regev A, Berho M, Jeffers LJ, et al. Sampling error and intraobserver variation in liver biopsy in patients with chronic HCV infection. Am J Gastroenterol. 2002;97:2614-8.

29. Persico M, Palmentieri B, Vecchione R, et al. Diagnosis of chronic liver disease: reproducibility and validation of liver biopsy. Am J Gastroenterol. 2002;97:491-2.

30. The French METAVIR Cooperative Study Group. Intraobserver and interobserver variations in liver biopsy interpretation in patients with chronic hepatitis C. Hepatology. 1994;20:15-20.

31. De Groote J, Desmet VJ, Gedigk P, et al. A classification of chronic hepatitis. Lancet. 1968;2:626-8.

32. Acute and chronic hepatitis revisited. Review by an international group. Lancet. 1977;29:914-9.

33. Knodell RG, Ishak KG, Black WC, Chen TS, Craig R, Kaplowitz $\mathrm{N}$, et al. Formulation and application of a numerical scoring system for assessing histological activity in asymptomatic chronic active hepatitis. Hepatology. 1981;1:431-5.

34. Bedossa P, Poynard T. An algorithm for the grading of activity in chronic hepatitis C. The METAVIR Cooperative Study Group. Hepatology. 1996;24:289-93.

35. Batts KP, Ludwig J. Chronic hepatitis. An update on terminology and reporting. Am J Surg Pathol. 1995;19:1409-17.

36. Hytiroglou P, Snover DC, Alves V, et al. Beyond "cirrhosis": a proposal from the International Liver Pathology Study Group. Am J Clin Pathol. 2012;137:5-9. 
37. Quaglia A, Alves VA, Balabaud C, et al. Role of aetiology in the progression, regression, and parenchymal remodelling of liver disease: implications for liver biopsy interpretation. Histopathology. 2016;68:953-67.

38. Garcia-Tsao G, Friedman S, Iredale J, et al. Now there are many (stages) where before there was one: In search of a pathophysiological classification of cirrhosis. Hepatology. 2010;51:1445-9.

39. Bedossa P. Reversibility of hepatitis B virus cirrhosis after therapy: who and why? Liver Int. 2015;35(Suppl 1):78-81.

40. Lim YS, Han S, Heo NY, et al. Mortality, liver transplantation, and hepatocellular carcinoma among patients with chronic hepatitis B treated with entecavir vs lamivudine. Gastroenterology. 2014;147:152-61.
41. Heimbach J, Kulik LM, Finn R, et al. AASLD guidelines for the treatment of hepatocellular carcinoma. Hepatology. 2017. https:// doi.org/10.1002/hep.29086.

42. Kim JH, Sinn DH, Kang W, et al. Low-level viremia and the increased risk of hepatocellular carcinoma in patients receiving entecavir treatment. Hepatology. 2016. https://doi.org/10.1002/ hep.28916.

43. Bruden DJT, McMahon BJ, Townshend-Bulson L, et al. Risk of end-stage liver disease, hepatocellular carcinoma, and liver-related death by fibrosis stage in the hepatitis C Alaska Cohort. Hepatology. 2017;66:37-45.

44. Papatheodoridis GV, Chan HL, Hansen BE, et al. Risk of hepatocellular carcinoma in chronic hepatitis B: assessment and modification with current antiviral therapy. J Hepatol. 2015;62:956-67. 